\title{
Interaction between interleukin-6 and angiotensin II receptor 1 in the hypothalamic paraventricular nucleus contributes to progression of heart failure
}

\author{
TAO WANG* ${ }^{*}$, WEN GAO* , KUN XIAO, QIANG LIU and RUYI JIA \\ Department of Cardiology, Medical School, Tai Shan Medical College, The Fourth \\ People's Hospital of Jinan, Jinan, Shandong 250031, P.R. China \\ Received January 27, 2016; Accepted January 16, 2017
}

DOI: $10.3892 / \mathrm{mmr} .2017 .6495$

\begin{abstract}
The association between interleukin-6 (IL-6) and angiotensin II receptor 1 (AT1-R) in modulating the progression of heart failure (HF) remains to be fully elucidated. The aim of the present study was to investigate the mechanism of IL-6 and AT1-R in a model of HF induced by surgery. Male Sprague-Dawley rats were randomly divided into five groups, including sham surgery and vehicle groups. The animals were treated for 4 weeks via paraventricular nucleus infusion with either vehicle, losartan (LOS; $200 \mu \mathrm{g} /$ day), IL-6 (1 $\mu \mathrm{g}$ /day) or LOS and IL-6 together (LOS+IL-6). The rats with HF had higher levels of IL-6, corticotropin-releasing hormone (CRH) and norepinephrine (NE), and a lower level of neuronal nitric oxide synthase (nNOS), compared with the rats in the sham surgery group. Treatment with LOS attenuated the decrease in nNOS and the increases in IL-6, CRH and NE; whereas treatment with IL-6 facilitated the lower expression of nNOS and higher expression levels of IL-6, CRH and NE. No differences in the expression levels of nNOS, CRH or NE were found between the LOS group and LOS+IL- 6 group. The results of the study demonstrated that IL-6 contributed to the progression of HF via the AT1-R pathway.
\end{abstract}

\section{Introduction}

As a prominent feature, heart failure (HF) is characterized by sympathetic hyperactivity (1). Following myocardial injury, increased sympathetic activity has been widely identified, even prior to the onset of HF (2). The association between

Correspondence to: Dr Ruyi Jia, Department of Cardiology, Medical School, Tai Shan Medical College, The Fourth People's Hospital of Jinan, 50 Shifan Road, Jinan, Shandong 250031, P.R. China E-mail: ruyijia126@126.com

*Contributed equally

Key words: heart failure, paraventricular nucleus, renin angiotensin system, angiotensin II receptor 1, interleukin-6, neuronal nitric oxide synthase inflammation and HF was investigated by Levine et al (3). However, there are few reports describing inflammation and $\mathrm{HF}$ in the paraventricular nucleus (PVN).

Sympathetic activity is widely regulated and includes the renin angiotensin system (RAS) (4). The RAS is important in the progression of HF. In the PVN, the inhibition of angiotensin receptor-type 1 (AT-1R) can improve sympathetic activity in HF (5). Further investigation has shown that increased cytokines in the PVN enhance the production of reactive oxygen species, which further exacerbates the sympathoexcitatory effects (6). In previous studies, the expression of several inflammatory cytokines, in addition to interleukin (IL)-1 $\beta$ (7), tumor necrosis factor- $\alpha$ (TNF- $\alpha$ ) (8) and IL-10 (9), have been reported to be altered in HF. IL-6 is the prototypal inflammatory cytokine and shows high expression in patients with HF $(10,11)$. The present study investigated the hypothesis that increased IL-6 contributes to the progression of HF via the AT1-R pathway.

Decreased neuronal nitric oxide synthase (nNOS) has also been identified in rats with $\mathrm{HF}(12,13)$. In addition, the antagonistic mechanism of nitric oxide (NO) is disrupted by angiotensin II-induced sympathetic hyperactivity, which then increases the production of superoxide, indicating the cross talk between the production of nNOS and the RAS mechanism (14-16). However, whether enhanced cytokine production and AT-1Rs in the PVN contribute to $\mathrm{HF}$ through regulating $\mathrm{nNOS}$ in rats remains to be fully elucidated. The present study also investigated alterations in nNOS in the PVN, including RAS-related hormones.

\section{Materials and methods}

Experimental animals. Male Sprague-Dawley rats (weight, 200-250 g; age, 6-8 weeks; $n=50$ ), were purchased from Shanghai Experimental Animal Breeding Co. (Shanghai, China). Following adaptation to the environment for 1 week, the rats were randomly divided into five groups: sham $(n=10)$, vehicle (VEH; $n=10)$, LOS $(n=10)$, LOS+IL-6 $(n=10)$ and IL-6 $(n=10)$. In the sham group, the rats received no treatment. The rats were housed in at $22 \pm 2^{\circ} \mathrm{C}$ and $40-60 \%$ humidity and light-controlled room $12 \mathrm{~h} \mathrm{light/dark}$ with free access to water. The experiments conformed to the Guide for the Care and Use of Laboratory Animals published by the US National Institutes of Health (17). 
General experimental protocol. Animals were anesthetized with intravenous infusion of sodium pentobarbital $\left[50 \mathrm{mg} / \mathrm{kg}^{-1}\right.$; intraperitoneal (i.p.)]. The rats underwent implantation of PVN cannulae, following which coronary artery ligation was performed to induce HF. At $24 \mathrm{~h}$ post-surgery, they were administered with artificial cerebrospinal fluid lasting for 4 weeks in the VEH group. At the same time, the rats were administered with AT1-R antagonist, LOS (200 $\mu \mathrm{g} /$ day $)$ in the LOS group ( $\mathrm{n}=10$; Merck Millipore; Darmstadt, Germany). The rats were administered with LOS (200 $\mu \mathrm{g} /$ day) and IL-6 $(1 \mu \mathrm{g} /$ day) in the LOS+IL-6 group (n=10; Sigma-Aldrich; Merck Millipore, Darmstadt, Germany). The rats were administered with IL-6 $(1 \mu \mathrm{g} / \mathrm{day})$ in the IL-6 group $(\mathrm{n}=10)$. Osmotic mini-pumps were implanted subcutaneously and connected with the cannulae for the continuous infusion of LOS, IL-6 and LOS+IL-6 or artificial cerebrospinal fluid directly into the PVN (0.1 ml/h pumping, once a day for 4 weeks). A stainless steel double cannula (Plastics One, Inc., Roanoke, VA, USA) was implanted into the PVN. Left ventricular (LV) function was assessed using echocardiography $24 \mathrm{~h}$ following recovery from surgery. At 4 weeks, the rats were anesthetized for echocardiograph examination and were then sacrificed by inducing an air embolism via intravenous infusion of $1 \mathrm{ml}$ air, tissue and plasma for further analyses was subsequently obtained.

Implantation of PVN cannulae and coronary ligation. Alzet miniosmotic pumps (model 2004; Durect Corporation, Cupertino, CA, USA) were used to enable the continuous infusion of drugs. The rats were placed into a stereotaxic apparatus following anesthesia (sodium pentobarbital; $50 \mathrm{mg} / \mathrm{kg}^{-1}$; i.p.) The method for PVN cannulation has been described previously (7). In brief, a stainless steel double cannula with a center-to-center distance of $0.5 \mathrm{~mm}$ was implanted into the PVN ( $2.0 \mathrm{~mm}$ posterior to the bregma and $8.5 \mathrm{~mm}$ ventral from the skull surface). HF was induced by ligation of the left anterior descending coronary artery, as previously described. In the sham group, surgery was performed in the same manner without ligating the coronary artery. Following surgery, the animals were administered with benzathine penicillin (30,000 units; IM).

Echocardiographic assessment of LV function. Echocardiography was performed using an Acuson Sequoia clinical imager (Siemens AG, Munich, Germany) fitted with an $8-\mathrm{MHz}$ sector-array probe, which generates 2-dimensional images at a rate of $100 / \mathrm{sec}$. The method for assessment LV function has been described previously (7). The animals were sedated with sodium pentobarbital $\left(25 \mathrm{mg} \mathrm{kg}^{-1}\right.$; i.p.) to facilitate positioning for echocardiographic examination. The animal was positioned in the left lateral recumbent position to optimize the windows for echocardiography. The anterior chest was shaved and pre-warmed acoustic coupling gel was applied. Short-axis images were acquired parallel to the mitral valve plane to obtain the largest cross-sectional image of the LV. Long-axis views were obtained perpendicular to the mitral valve plane. Images were stored for subsequent offline analysis. LV end-diastolic volume (LVEDV), LV end-systolic volume, LV ejection fraction (LVEF), LV stroke volume and $\mathrm{LV}$ mass were computed. The region of the $\mathrm{LV}$, which exhibited akinesis was planimetered electronically and expressed as a percentage of the total LV silhouette to estimate the size of the ischemic zone. Only animals with large infarctions (ischemic zone $\geq 40 \%$ ) were used in the examination.

Tissue microdissection. The animals were sacrificed by decapitation, and their brains were rapidly removed and frozen on dry ice. Serial sections $(300 \mathrm{~mm})$ of the brain were obtained using a cryostat maintained at $-10^{\circ} \mathrm{C}$. The sections were transferred to coverslips, which were placed on a cold stage set at $-10^{\circ} \mathrm{C}$. The PVN was microdissected out using Palkovits' microdissection technique (18).

Immunohistochemistry. The method for immunohistochemistry has been previously described (19). Paraffin sections of the artery were deparaffinized and endogenous peroxidase activity was inactivated with $3 \% \mathrm{H}_{2} \mathrm{O}_{2}$ for $10 \mathrm{~min}$. The Fra-like (Fra-LI) primary antibody (1:200; cat. no. sc-271657; Santa Cruz Biotechnology, Inc., Dallas, TX, USA) or normal blocking serum was added and incubated overnight. Biotin-conjugated goat anti-mouse immunoglobulin $\mathrm{G}(\operatorname{IgG})$ (1:1,000; cat. no. 115-035-003; Jackson, ImmunoResearch Laboratories, Inc., West Grove, PA, USA) was used as the secondary antibody and incubated for $30 \mathrm{~min}$ at $4^{\circ} \mathrm{C}$. An avidin-biotin enzyme reagent was sequentially added and incubated for $20 \mathrm{~min}$. A peroxidase substrate was added and incubated until desired stain intensity developed. Finally, the sections were covered with a glass cover slip and observed under a light microscope. The intensity of positive staining in tissue was analyzed by integrated optical density (IOD) using Image-Pro Plus software (Media Cybernetics, Rockville, MD, USA). The Fra-LI expression was expressed as (IOD/area)x100 in accordance with a previous study (20).

$R N A$ extraction and reverse transcription-quantitative polymerase chain reaction ( $R T-q P C R)$ analysis. The method for mRNA analysis has been described previously (19). Total RNA was extracted from the artery specimens using TRIzol ${ }^{\circledR}$ reagent according to the manufacturer's protocol (Invitrogen; Thermo Fisher Scientific, Inc., Waltham, MA, USA). The total RNA $(1 \mu \mathrm{g})$ was used as a template to produce cDNA using an RT kit (BioDev-Tech Co., Ltd., Beijing, China). The qPCR was performed by monitoring the increase in fluorescence of the SYBR Green dye using GreenMaster mix (Genaxxon BioScience, Ulm, Germany) according to the manufacturer's protocol. The primer sets used to amplify nNOS were 5'-gccatccagcataatgacccag-3' (sense) and 5'-gagggtgatccaaagatgtcc tc $-3^{\prime}$ (antisense) (21). The primer sets used to amplify CRH were 5'-cagaacaacagtgcgggctca-3' (sense) and 5'-aaggcagacagggcgaca gag-3' (antisense) (22). The primer sets used to amplify GAPDH were 5'-ccactttgtgaagctcatttcct-3' (sense) and 5'-tcgtcctcctctggtgctct-3' (antisense). PCR amplification was performed with tag polymerase for 32 cycles at $95^{\circ} \mathrm{C}$ for $45 \mathrm{sec}, 62^{\circ} \mathrm{C}$ for $30 \mathrm{sec}$ and $72^{\circ} \mathrm{C}$ for $1 \mathrm{~min}$ (for CRH, nNOS and GAPDH). The $2^{-\Delta \Delta \mathrm{Cq}}$ method (23) was used to determine relative changes in the gene expression of $\mathrm{CRH}, \mathrm{nNOS}$ and GAPDH. Values are expressed in relative quantities to the mRNA expression in the control group.

Western blot analysis. The method for western blot analysis has been described previously (19). The specimens were washed with ice-cold PB and lysed for $20 \mathrm{~min}$ on ice with 
Table I. Echocardiograph measures for evaluation of LV function.

\begin{tabular}{|c|c|c|c|c|c|}
\hline Measurement & SHAM & VEH & LOS & LOS+IL-6 & IL-6 \\
\hline \multicolumn{6}{|l|}{$24 \mathrm{~h}$} \\
\hline $\mathrm{IZ}(\%)$ & - & $47 \pm 5$ & $46 \pm 5$ & $46 \pm 4$ & $46 \pm 7$ \\
\hline LVEF & $0.78 \pm 0.04$ & $0.38 \pm 0.04$ & $0.37 \pm 0.05$ & $0.37 \pm 0.06$ & $0.36 \pm 0.05$ \\
\hline LVEDV & $0.35 \pm 0.03$ & $0.75 \pm 0.05$ & $0.77 \pm 0.07$ & $0.78 \pm 0.06$ & $0.76 \pm 0.08$ \\
\hline LVEDV/mass & $0.53 \pm 0.05$ & $1.12 \pm 0.08$ & $1.09 \pm 0.08$ & $1.06 \pm 0.07$ & $1.08 \pm 0.05$ \\
\hline \multicolumn{6}{|l|}{4 weeks } \\
\hline IZ (\%) & - & $48 \pm 6$ & $47 \pm 4$ & $47 \pm 4$ & $47 \pm 5$ \\
\hline LVEF & $0.79 \pm 0.07$ & $0.32 \pm 0.05$ & $0.42 \pm 0.06$ & $0.41 \pm 0.05$ & $0.26 \pm 0.05$ \\
\hline LVEDV & $0.36 \pm 0.05$ & $1.41 \pm 0.04$ & $1.05 \pm 0.05$ & $1.06 \pm 0.04$ & $1.66 \pm 0.05$ \\
\hline LVEDV/mass & $0.54 \pm 0.05$ & $1.74 \pm 0.05$ & $1.24 \pm 0.05$ & $1.22 \pm 0.06$ & $1.96 \pm 0.06$ \\
\hline
\end{tabular}

Values are presented as the mean \pm standard error of the mean. SHAM, sham surgery; VEH, vehicle; LOS, losartan; IL-6 interleukin-6; LOS+IL-6; IZ, infarction zone; LVEDV, left ventricular end diastolic volume.
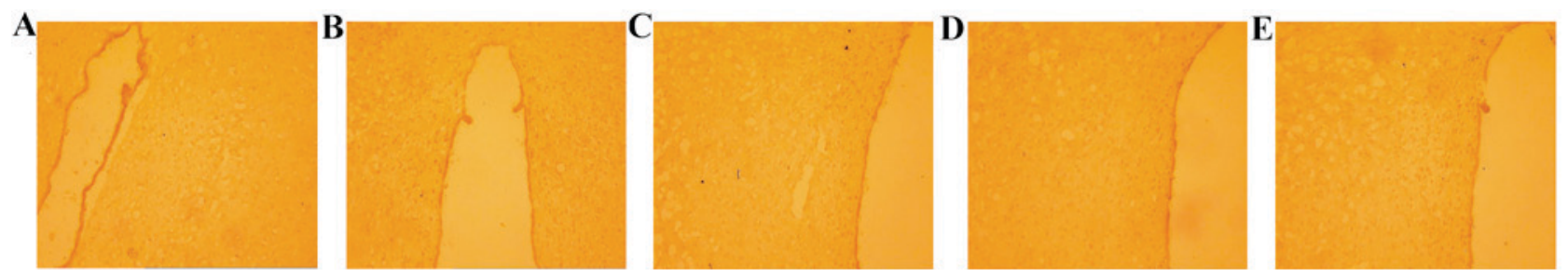

Figure 1. Immunohistochemical analysis of Fra-like activity in the paraventricular nucleus of the hypothalamus (magnification, x200). (A) Sham group; (B) vehicle group; (C) LOS group; (D) LOS+IL-6 group; (E) IL-6 group. LOS, losartan; IL-6, interleukin-6.

lysis buffer (cat.no. SC-003; Invent, Lund, Sweden). Following lysis, the lysates were centrifuged for $4 \mathrm{~min}$ at $394 \mathrm{x} g$ and the supernatants were collected in a fresh tube on ice. The protein concentrations in each sample were determined using a BCA assay. Total proteins $(100 \mu \mathrm{g})$ were mixed with loading buffer with the anionic denaturing detergent, sodium dodecyl sulfate (SDS), boiled for $5 \mathrm{~min}$ and then resolved by $10 \%$ SDS polyacrylamide gel electrophoresis. The proteins were transferred onto PVDF membranes. Following blocking of the membranes in TBST containing non-fat milk for $1 \mathrm{~h}$ at $4^{\circ} \mathrm{C}$ under agitation, the membranes were washed three times in TBST and incubated for $2 \mathrm{~h}$ at $4^{\circ} \mathrm{C}$ with anti-rat nNOS antibody (1:200 dilution; cat. no. NB120-3511; Novus Biologicals, Ltd., Cambridge, UK), anti-rat CRH antibody (1:200 dilution; cat. no. NBP1-42614; Novus Biologicals, Ltd.) or GAPDH monoclonal antibody (1:200 dilution; cat. no. NB100-56875; Novus Biologicals, Ltd.). Following washing three times in TBST, the membranes were incubated with HRP-conjugated goat anti-rabbit IgG $(1: 1,000$; cat. no. AB501-01A; Novoprotein, Shanghai, China) at temperature for $1 \mathrm{~h}$ at $4^{\circ} \mathrm{C}$ and then washed three times with TBST. The immunobands were detected using a streptavidin amplification reagent (cat. no. WBKL SOO 50; EMD Millipore, Billerica, MA, USA) according to the manufacturer's protocol.

ELISA for IL- 6 and NE. The method for the analysis of IL-6 and NE in plasma has been previously described (19). From all animals, fresh blood samples $(3 \mathrm{ml})$ were obtained via the femoral vein and centrifuged at 2,465 $\mathrm{x} \mathrm{g}$ for $10 \mathrm{~min}$ at $4^{\circ} \mathrm{C}$. The supernatant was placed in a clean centrifuge tube and frozen at $-20^{\circ} \mathrm{C}$. Plasma concentrations of IL- 6 and NE were assayed using ELISA kits (R\&D Systems, Inc., Minneapolis, MN, USA) according to the manufacturer's protocol. The minimum detectable concentration of the kits was $<1.0 \mathrm{pg} / \mathrm{ml}$; the variation in different boards was $<15 \%$. They were not cross-reactive with other soluble structural analogues.

Statistical analysis. The data were analyzed using the SPSS 11.5 program (SPSS, Inc., Chicago, IL, USA) for Windows. Quantitative data are presented as the mean \pm standard deviation. For comparison between multiple groups, data was analyzed using one-way analysis of variance, and with Student-Newman-Keuls post hoc analysis. $\mathrm{P}<0.05$ was considered to indicate a statistically significant difference.

\section{Results}

$L V$ function alterations. Echocardiography was used to evaluate alterations in LV function (Table I). At $24 \mathrm{~h}$, the LVEDV, LVEDV/mass ratio and \% infarction zone (IZ) were higher in the VEH, LOS, IL-6, and LOS+IL-6 groups, compared with those in the sham group. No differences were observed in these parameters among the HF rats assigned to the LOS, IL-6, LOS+IL-6 or the VEH treatment groups at $24 \mathrm{~h}$ $(\mathrm{P}>0.05)$. At 4 weeks, LVEDV and LVEDV/mass ratio were higher, compared with the 24-h baseline values in the LOS, LOS+IL-6 and vehicle-treated HF rats $(\mathrm{P}<0.01)$. At 4 weeks, LVEF was higher in the HF rats in the LOS and LOS+IL-6 treatment groups, compared with the HF rats in the VEH or 


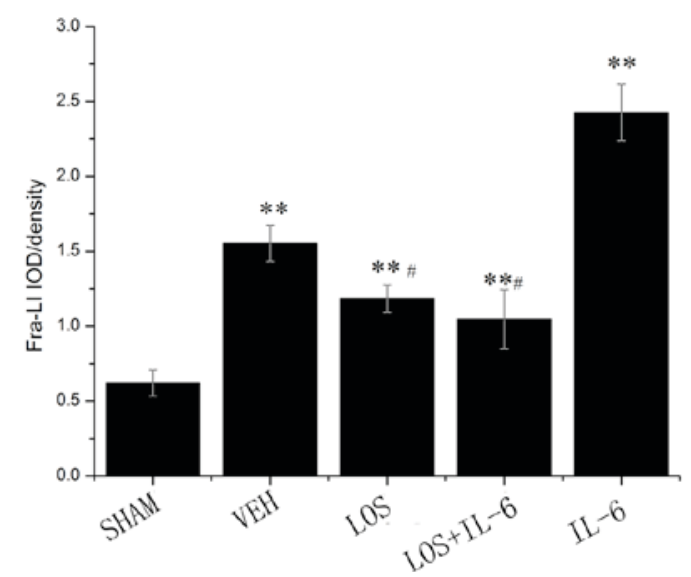

Figure 2. Fra-like activity immunohistochemical staining in the paraventricular nucleus of the hypothalamus. ${ }^{* *} \mathrm{P}<0.01$, vs. sham group; ${ }^{\#} \mathrm{P}>0.05$, LOS group vs. LOS+IL-6 group. SHAM, sham surgery; VEH, vehicle; LOS, losartan; IL-6, interleukin-6.
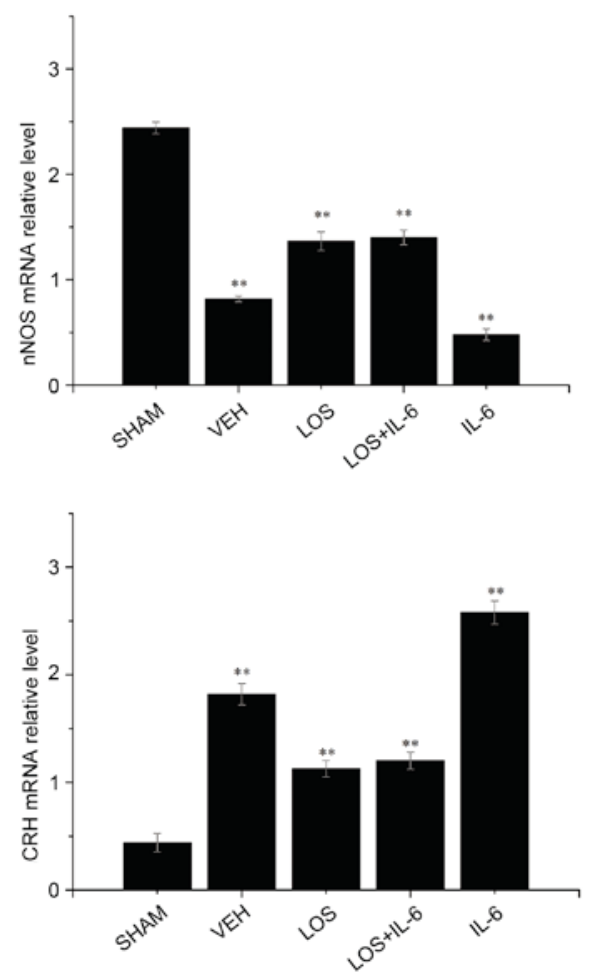

Figure 3. Gene expression of nNOS and $\mathrm{CRH}$ in the hypothalamus. ${ }^{* *} \mathrm{P}<0.01$, vs. sham group. SHAM, sham surgery; VEH, vehicle; LOS, losartan; IL-6, interleukin-6; nNOS, neuronal nitric oxide synthase; $\mathrm{CRH}$, corticotropin-releasing hormone.

IL-6 treatment groups $(\mathrm{P}<0.01)$. The LVEDV and LVEDV/mass in the HF rats, which received LOS or LOS+IL-6 were lower, compared with those in the rats in the VEH and IL-6 treatment groups $(\mathrm{P}<0.01)$, whereas no significant difference were observed between the LOS and LOS+IL-6 groups $(\mathrm{P}>0.05)$.

Location and expression of Fra-LI activity. The immunohistochemistry showed that the staining intensity of Fra-LI was minimal in the sham group (Fig. 1). Fra-LI activity is an indicator of chronic neuronal excitation. Lower levels of Fra-LI immunostaining were detected in the LOS and LOS+IL-6 groups, compared with those in the VEH and IL-6 groups $(\mathrm{P}<0.01)$. The HF rats treated with IL-6 had increased Fra-LI-positive PVN neurons, compared with those in the VEH, LOS and LOS+IL-6 groups $(\mathrm{P}<0.01)$. No significant differences were observed between the LOS and LOS+IL-6 groups (P>0.05; Fig. 2).

nNOS gene expression. In order to investigate the mechanism responsible for alterations in the HF mice, mRNA levels of nNOS were analyzed using RT-qPCR analysis (Fig. 3). Compared with the VEH group, the mRNA expression levels of nNOS were significantly increased in the sham group $(\mathrm{P}<0.01)$, LOS group $(\mathrm{P}<0.05)$ and LOS+IL-6 group $(\mathrm{P}<0.05)$. The HF rats treated with IL-6 had fewer nNOS-positive neurons, compared with the rats treated with LOS+IL-6 $(\mathrm{P}<0.01)$. No significant difference the expression of $\mathrm{nNOS}$ in PVN neurons was observed between the LOS group and LOS+IL-6 group $(\mathrm{P}>0.05)$.

Gene expression of $C R H$. Compared with the sham group, the mRNA expression of CRH was significantly increased in the LOS group, LOS+IL-6 group and VEH group $(\mathrm{P}<0.01)$. By contrast, the mRNA expression levels of CRH were decreased in the LOS and LOS+IL-6 groups, compared with that in the IL-6 group $(\mathrm{P}<0.01)$. The rats treated with IL-6 had a higher number of $\mathrm{CRH}$-positive neurons, compared with the rats treated with LOS+IL-6 or VEH $(\mathrm{P}<0.01)$. No significant difference in the gene expression of $\mathrm{CRH}$ in PVN neurons was observed between the LOS group and LOS+IL-6 group (P>0.05; Fig. 3).

Protein expression of $n N O S$. Western blot analysis was used to examine whether the protein levels of nNOS correlated with alterations in its mRNA levels (Fig. 4). Compared with the VEH group and IL-6 group, significant increases in the protein expression of nNOS were observed in the sham group, LOS group and LOS+IL-6 group in the blots $(\mathrm{P}<0.01)$. The rats treated with IL-6 exhibited lower expression of nNOS, compared with the rats treated with LOS+IL-6 $(\mathrm{P}<0.05)$. No differences in the protein expression of nNOS were found between the LOS group and LOS+IL-6 group $(\mathrm{P}>0.05)$.

Protein expression of $\mathrm{CRH}$. Western blot analysis was used to examine whether the protein levels of $\mathrm{CRH}$ were correlated with alterations in its mRNA levels (Fig. 4). Compared with the sham group, significant increases in the expression levels of CRH were observed in the VEH group, LOS group, LOS+IL-6 group and IL- 6 group in the blots $(\mathrm{P}<0.01)$. The rats treated with IL-6 exhibited higher expression of $\mathrm{CRH}$, compared with the rats treated with LOS+IL-6 $(\mathrm{P}<0.01)$. No difference in the protein expression of CRH was observed between the LOS group and LOS+IL-6 group $(\mathrm{P}>0.05)$.

Serum levels of IL-6 and NE. Compared with the sham group, the levels of IL-6 and NE were significantly increased following treatment $(\mathrm{P}<0.01$; Fig. 5). Compared with the VEH group, treatment with LOS and LOS+IL-6 significantly decreased the expression of IL-6 and NE $(\mathrm{P}<0.01)$, and treatment with IL-6 


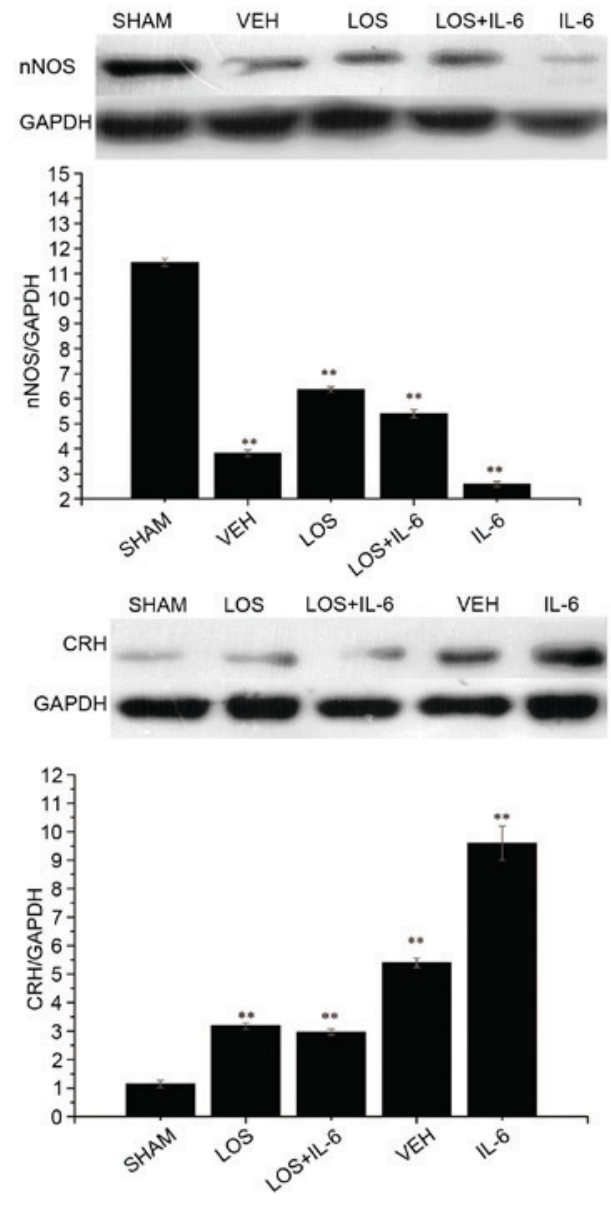

Figure 4. Western blot analysis of the protein expression of nNOS and CRH in the hypothalamus. ${ }^{* *} \mathrm{P}<0.01$, vs. Sham group. SHAM, sham surgery; VEH, vehicle; LOS, losartan; IL-6, interleukin-6; nNOS, neuronal nitric oxide synthase; $\mathrm{CRH}$, corticotropin-releasing hormone.

increased the levels of IL-6 and NE $(\mathrm{P}<0.05)$. No significant differences were observed in the levels of IL- 6 or NE between the LOS group and LOS+IL-6 group ( $\mathrm{P}<0.05$; Fig. 5).

\section{Discussion}

Despite modern advances in technology, HF remains a leading contributor to morbidity and mortality rates worldwide (24). Sympathoexcitation is a pathophysiological hallmark of HF (25). Studies have indicated that excitation is negatively correlated with the prognosis of $\operatorname{HF}(26,27)$. However, the precise mechanism underlying HF remains to be fully elucidated. In the present study, it was found that IL-6 was associated with AT1-R in rats with HF. Treatment with LOS led to increased nNOS, decreased CRH and NE, and improved heart function. The effect of IL-6 was opposite to that of LOS in $\mathrm{HF}$, and treatment with LOS reduced the damaging effects.

It has been reported that enhanced sympathetic nerve activity (SNA) and inflammatory cytokines lead to severe ventricular arrhythmias, which is the major contributor to rates of mortality among patients with $\operatorname{HF}(28,29)$. The increase in SNA in HF is due to an imbalance between inhibitory and excitatory mechanisms within specific areas in the central nervous system (CNS), including the PVN of the hypothalamus (30). SNA to the kidneys results in renal
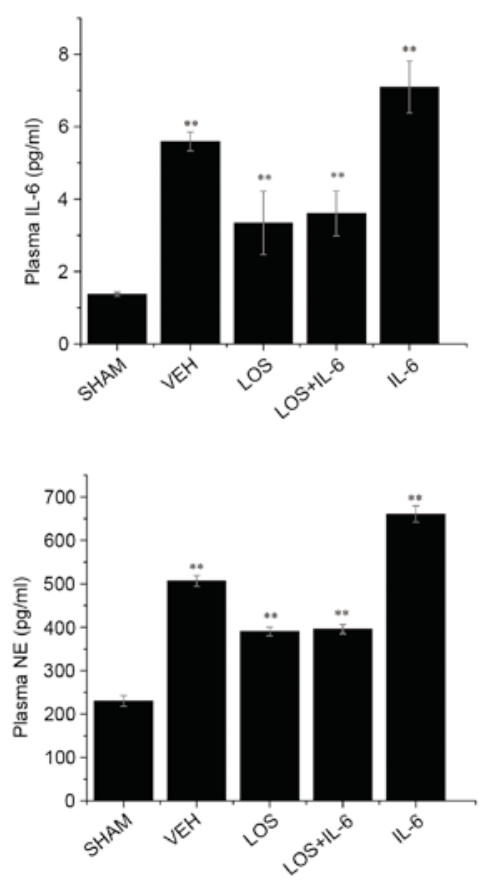

Figure 5. Serum levels of NE and IL-6. ${ }^{* *} \mathrm{P}<0.01$, vs. Sham group. SHAM, sham surgery; VEH, vehicle; LOS, losartan; NE, norepinephrine; IL-6, interleukin-6.

vasoconstriction, increased renal sodium retention and increased renin release, and consequently to elevated levels of angiotensin II and aldosterone (31). An increasing number of experimental studies have suggested that PVN is key in the progression of HF (26). Studies have found that $\mathrm{CRH}$ and $\mathrm{NE}$ contribute to sympathoexcitation in rats with ischemia-induced HF $(32,33)$. The results of the present study are consistent with these previous studies. LOS, as an angiotensin receptor inhibitor, has been used in clinical management for several years (34); it decreases plasma renin activity and inhibits the conversion of angiotensinogen to angiotensin I. In the present study, it was found that IL-6 stimulated the expression of CRH and NE, and led to worsening heart function. The microinjection of IL-6 and LOS in combination attenuated the effects of IL-6. Taken together, the results indicated that IL-6 increased sympathetic activity to simulate the progression of HF via the AT1-R pathway.

Previous studies have suggested that repressing the production of cytokines improves sympathetic activity in rats with HF (35). For example, it was found that the production of TNF- $\alpha$ and IL-1 $\beta$ leads to abnormal LV dysfunction and remodeling in the progression of $\mathrm{HF}(7,36,37)$. IL- 6 is known to regulate inflammatory reactions, immunity and neural development $(38,39)$. Higher expression levels of IL-6 in plasma and cardiac tissue directly correlate with HF $(40,41)$. There are few reports describing the association between IL-6 and PVN in HF, although IL-6 receptor is increased in the PVN of rats with HF (42). In the present study, it was also found that plasma levels of IL- 6 were elevated in HF rats, which correlated with the severity of heart function. In accordance with these findings, the present study identified that treatment with LOS improved cytokine-induced diastolic and systolic dysfunction in HF rats. Su et al (43) revealed 
that AT1-R inhibitor significantly reduces the level of IL-6 in macrophages. These data indicate the cross talk between cytokines and AT1-R in HF. In the present study, treatment with IL-6 deteriorated heart function, whereas the AT1-R inhibitor alleviated the dysfunction induced by IL- 6 . This suggested that AT1-R may be a target site for IL-6 in PVN.

In addition to interaction with cytokines, AT1-R inhibitor has also been reported to interact with $\mathrm{NO}$ in the PVN of animals with HF. NO is a gaseous neuromodulator substance, which is key in the regulation of sympathetic tone $(44,45)$ and protection of endothelial function (46). However, the role of nNOS in the pathophysiology of HF remains to be fully elucidated. The reduced production of NO may lead to amplification of the angiotensin II signal and enhance sympathetic activity (47). In the neurons of the PVN, nNOS exerts an important function in controlling central sympathetic outflow and enhancing sympathetic activity in HF (48). In the present study, it was found that LOS significantly increased the expression of nNOS and improved LV function, whereas IL-6 had adverse effects. Therefore, in addition to inhibiting SNA, LOS increased the expression of nNOS, which delayed the process of HF.

Taken together, the results of the present study demonstrated that the interaction between AT1-R and IL-6 is important in $\mathrm{HF}$, and treatment with LOS significantly attenuated the effects caused by IL-6, which was accompanied by imbalance in sympathoexcitation and nNOS in the PVN.

\section{Acknowledgements}

This study was supported by the Jinan Science and Technology Project (grant no. 201201051) and Shandong Provincial Natural Science Foundation, China (grant no. ZR2014HP021).

\section{References}

1. Francis GS, Benedict C, Johnstone DE, Kirlin PC, Nicklas J, Liang CS, Kubo SH, Rudin-Toretsky E and Yusuf S: Comparison of neuroendocrine activation in patients with left ventricular dysfunction with and without congestive heart failure. A substudy of the studies of left ventricular dysfunction (SOLVD). circulation 82: 1724-1729, 1990.

2. Zhang ZH, Francis J, Weiss RM and Felder RB: The renin-angiotensin-aldosterone system excites hypothalamic paraventricular nucleus neurons in heart failure. Am J Physiol Heart Circ Physiol 283: H423-H433, 2002.

3. Levine B, Kalman J, Mayer L, Fillit HM and Packer M: Elevated circulating levels of tumor necrosis factor in severe chronic heart failure. N Engl J Med 323: 236-241, 1990.

4. Francis J, Chu Y, Johnson AK, Weiss RM and Felder RB Acute myocardial infarction induces hypothalamic cytokine synthesis. Am J Physiol Heart Circ Physiol 286: H2264-H2271, 2004.

5. Guggilam A, Haque M, Kerut EK, McIlwain E, Lucchesi P, Seghal I and Francis J: TNF-alpha blockade decreases oxidative stress in the paraventricular nucleus and attenuates sympathoexcitation in heart failure rats. Am J Physiol Heart Circ Physiol 293 H599-H609, 2007.

6. Dawson VL and Dawson TM: Nitric oxide neurotoxicity. J Chem Neuroanat 10: 179-190, 1996

7. Liu Q, Wang T, Yu H, Liu B and Jia R: Interaction between interleukin-1 beta and angiotensin II receptor 1 in hypothalamic paraventricular nucleus contributes to progression of heart failure. J Interferon Cytokine Res 34: 870-875, 2014.

8. Wei P, Yang XJ, Fu Q, Han B, Ling L, Bai J, Zong B and Jiang CY: Intermedin attenuates myocardial infarction through activation of autophagy in a rat model of ischemic heart failure via both cAMP and MAPK/ERK1/2 pathways. Int J Clin Exp Pathol 8: 9836-9844, 2015.
9. Duan HY, Liu DM, Qian P, Wang SL, Yan LJ, Wu JT, Yang HT, Fan XW and Chu YJ: Effect of atorvastatin on plasma NT-proBNP and inflammatory cytokine expression in patients with heart failure. Genet Mol Res 14: 15739-15748, 2015.

10. Demissei BG, Valente MA, Cleland JG, O'Connor CM, Metra M, Ponikowski P, Teerlink JR, Cotter G, Davison B, Givertz MM, et al: Optimizing clinical use of biomarkers in high-risk acute heart failure patients. Eur J Heart Fail 18: 269-280, 2016.

11. Bielecka-Dabrowa A, von Haehling S, Aronow WS, Ahmed MI, Rysz $\mathrm{J}$ and Banach $\mathrm{M}$ : Heart failure biomarkers in patients with dilated cardiomyopathy. Int J Cardiol 168: 2404-2410, 2013.

12. Patel KP, Zhang K, Zucker IH and Krukoff TL: Decreased gene expression of neuronal nitric oxide synthase in hypothalamus and brainstem of rats in heart failure. Brain Res 734: 109-115, 1996.

13. Li BF, Liu YF, Cheng Y, Zhang KZ, Li TM and Zhao N: Protective effect of inducible nitric oxide synthase inhibitor on pancreas transplantation in rats. World J Gastroenterol 13: 6066-6071, 2007.

14. Rajagopalan S, Kurz S, Münzel T, Tarpey M, Freeman BA, Griendling KK and Harrison DG: Angiotensin II-mediated hypertension in the rat increases vascular superoxide production via membrane NADH/NADPH oxidase activation. Contribution to alterations of vasomotor tone. J Clin Invest 97: 1916-1923, 1996.

15. Jang JH, Chun JN, Godo S, Wu G, Shimokawa H, Jin CZ, Jeon JH, Kim SJ, Jin ZH and Zhang YH: ROS and endothelial nitric oxide synthase (eNOS)-dependent trafficking of angiotensin II type 2 receptor begets neuronal NOS in cardiac myocytes. Basic Res Cardiol 110: 21, 2015.

16. Ratliff BB, Sekulic M, Rodebaugh J and Solhaug MJ: Angiotensin II regulates NOS expression in afferent arterioles of the developing porcine kidney. Pediatr Res 68: 29-34, 2010.

17. National Research Council (US) Institute for Laboratory Animal Research: Guide for the Care and Use of Laboratory Animals. Washington (DC): National Academies Press (US); 1996.

18. Mohan Kumar SM, Mohan Kumar PS and Quadri SK: Specificity of interleukin-1beta-induced changes in monoamine concentrations in hypothalamic nuclei: Blockade by interleukin-1 receptor antagonist. Brain Res Bull 47: 29-34, 1998.

19. Cao H, Wen G and Li H: Role of peroxisome proliferator-activated receptor $\alpha$ in atherosclerosis. Mol Med Rep 9: 1755-1760, 2014.

20. Campioli E, Batarseh A, Li J and Papadopoulos V: The endocrine disruptor mono-(2-ethylhexyl) phthalate affects the differentiation of human liposarcoma cells (SW 872). PLoS One 6: e28750, 2011.

21. Sousa LE, Magalhães WG, Bezerra FS, Santos RA, Campagnole-Santos MJ, Isoldi MC and Alzamora AC: Exercise training restores oxidative stress and nitric oxide synthases in the rostral ventrolateral medulla of renovascular hypertensive rats. Free Radic Res 49: 1335-1343, 2015.

22. Ge JF, Xu YY, Qin G, Peng YN, Zhang CF, Liu XR, Liang LC, Wang ZZ and Chen FH: Depression-like behavior induced by Nesfatin-1 in rats: Involvement of increased immune activation and imbalance of synaptic vesicle proteins. Front Neurosci 9: $429,2015$.

23. Livak KJ and Schmittgen TD: Analysis of relative gene expression data using real-time quantitative PCR and the 2(-Delta Delta C(T)) Method. Methods 25: 402-408, 2001.

24. Roger VL, Go AS, Lloyd-Jones DM, Adams RJ, Berry JD, Brown TM, Carnethon MR, Dai S, de Simone G, Ford ES, et al: Heart disease and stroke statistics-2011 update: A report from the American Heart Association. Circulation 123: e18-e29, 2011.

25. Watson AM, Hood SG and May CN: Mechanisms of sympathetic activation in heart failure. Clin Exp Pharmacol Physiol 33: 1269-1274, 2006

26. Floras JS: Sympathetic activation in human heart failure: dIverse mechanisms, therapeutic opportunities. Acta Physiol Scand 177: 391-398, 2003.

27. Gao L, Wang W, Liu D and Zucker IH: Exercise training normalizes sympathetic outflow by central antioxidant mechanisms in rabbits with pacing-induced chronic heart failure. Circulation 115: 3095-3102, 2007.

28. Aronson D and Burger AJ: Concomitant beta-blocker therapy is associated with a lower occurrence of ventricular arrhythmias in patients with decompensated heart failure. J Card Fail 8: 79-85, 2002.

29. Kowalewski M, Urban M, Mroczko B and Szmitkowski M: Proinflammatory cytokines (IL-6, TNF-alpha) and cardiac troponin I (cTnI) in serum of young people with ventricular arrhythmias. Pol Arch Med Wewn 108: 647-651, 2002 (In Polish). 
30. Kang YM, Yang Q, Yu XJ, Qi J, Zhang Y, Li HB, Su Q and Zhu GQ: Hypothalamic paraventricular nucleus activation contributes to neurohumoral excitation in rats with heart failure. Regen Med Res 2: 2, 2014.

31. Ramchandra R and Barrett CJ: Regulation of the renal sympathetic nerves in heart failure. Front Physiol 6: 238, 2015

32. Kang YM, Zhang AQ, Zhao XF, Cardinale JP, Elks C, Cao XM Zhang ZW and Francis J: Paraventricular nucleus corticotrophin releasing hormone contributes to sympathoexcitation via interaction with neurotransmitters in heart failure. Basic Res Cardiol 106: 473-483, 2011.

33. Yildiz M, Hasdemir H, Turkkan C, Astarcioglu MA, Alper AT, Sahin A and Ozkan M: Acute effects of cardiac resynchronization therapy on arterial distensibility and serum norepinephrine levels in advanced heart failure. Cardiol J 20: 304-309, 2013.

34. Timmermans PB, Duncia JV, Carini DJ, Chiu AT, Wong PC, Wexler RR and Smith RD: Discovery of losartan, the first angiotensin II receptor antagonist. J Hum Hypertens 9 (Suppl 5): S3-S18, 1995.

35. Wang Y, Patel KP, Cornish KG, Channon KM and Zucker IH: nNOS gene transfer to RVLM improves baroreflex function in rats with chronic heart failure. Am J Physiol Heart Circ Physiol 285: H1660-H1667, 2003.

36. Mann DL: Inflammatory mediators and the failing heart: Past, present, and the foreseeable future. Circ Res 91: 988-998, 2002.

37. Anker SD and von Haehling S: Inflammatory mediators in chronic heart failure: An overview. Heart 90: 464-470, 2004.

38. Romano M, Sironi M, Toniatti C, Polentarutti N, Fruscella P, Ghezzi P, Faggioni R, Luini W, van Hinsbergh V, Sozzani S, et al: Role of IL-6 and its soluble receptor in induction of chemokines and leukocyte recruitment. Immunity 6: 315-325, 1997.

39. Marshall LF: Head injury: Recent past, present, and future. Neurosurgery 47: 546-561, 2000

40. Deten A, Volz HC, Briest W and Zimmer HG: Cardiac cytokine expression is upregulated in the acute phase after myocardia infarction. Experimental studies in rats. Cardiovasc Res 55 329-340, 2002.
41. Torre-Amione G, Kapadia S, Benedict C, Oral H, Young JB and Mann DL: Proinflammatory cytokine levels in patients with depressed left ventricular ejection fraction: A report from the studies of left ventricular dysfunction (SOLVD). J Am Coll Cardiol 27: 1201-1206, 1996.

42. Helwig BG, Musch T, Craig RA and Kenney MJ: Increased interleukin- 6 receptor expression in the paraventricular nucleus of rats with heart failure. Am J Physiol Regul Integr Comp Physiol 292: R1165-1173, 2007.

43. Su Q, Liu JJ, Cui W, Shi XL, Guo J, Li HB, Huo CJ, Miao YW, Zhang M, Yang Q and Kang YM: Alpha lipoic acid supplementation attenuates reactive oxygen species in hypothalamic paraventricular nucleus and sympathoexcitation in high salt-induced hypertension. Toxicol Lett 241: 152-158, 2016.

44. Stojanović M, Šćepanović L, Hrnčić D, Rašić-Marković A, Djuric D and Stanojlović O: Multidisciplinary approach to nitric oxide signaling: Focus on the gastrointestinal and the central nervous system. Vojnosanitetski Pregled 72: 619-624, 2015.

45. Cheng WH, Lu PJ, Ho WY, Tung CS, Cheng PW, Hsiao M and Tseng CJ: Angiotensin II inhibits neuronal nitric oxide synthase activation through the ERK1/2-RSK signaling pathway to modulate central control of blood pressure. Circ Res 106: 788-795, 2010.

46. Capettini LS, Cortes SF, Silva JF, Alvarez-Leite JI and Lemos VS: Decreased production of neuronal NOS-derived hydrogen peroxide contributes to endothelial dysfunction in atherosclerosis. Br J Pharmacol 164: 1738-1748, 2011.

47. Liu JL, Murakami H and Zucker IH: Angiotensin II-nitric oxide interaction on sympathetic outflow in conscious rabbits. Circ Res 82: 496-502, 1998.

48. Zhang K, Zucker IH and Patel KP: Altered number of diaphorase (NOS) positive neurons in the hypothalamus of rats with heart failure. Brain Res 786: 219-225, 1998. 